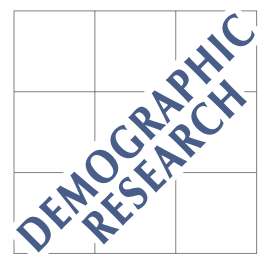

Demographic Research a free, expedited, online journal of peer-reviewed research and commentary in the population sciences published by the Max Planck Institute for Demographic Research Konrad-Zuse Str. 1, D-18057 Rostock · GERMANY www.demographic-research.org

DEMOGRAPHIC RESEARCH

VOLUME 21, ARTICLE 5, PAGES 109-134 PUBLISHED 07 AUGUST 2009

http://www.demographic-research.org/Volumes/Vol21/5/

DOI: $\quad$ 10.4054/DemRes.2009.21.5

Research Article

\title{
An analysis of life expectancy and economic production using expectile frontier zones
}

\author{
Sabine K. Schnabel
}

Paul H.C. Eilers

(C) 2009 Sabine K. Schnabel \& Paul H.C. Eilers.

This open-access work is published under the terms of the Creative Commons Attribution NonCommercial License 2.0 Germany, which permits use, reproduction \& distribution in any medium for non-commercial purposes, provided the original author(s) and source are given credit. See http://creativecommons.org/licenses/by-nc/2.0/de/ 


\section{Table of Contents}

1 Introduction $\quad 110$

2 An overview of previous research 111

2.1 Life expectancy and GDP 112

$2.2 \quad$ Models for frontier estimation 113

2.2.1 Quantile regression 113

$\begin{array}{ll}\text { 2.2.2 } & \text { Econometric methods for efficiency estimation } \\ 2.313\end{array}$

$\begin{array}{lll}2.3 & \text { Data description } & 114\end{array}$

3 Least Asymmetrically Weighted Squares Smoothing 116

$\begin{array}{ll}3.1 & \text { LAWS in a nutshell } \\ 3.217\end{array}$

$\begin{array}{lll}3.2 & \text { Extreme observations } & 119\end{array}$

$\begin{array}{lll}3.3 & \text { Theoretical expectiles } & 119\end{array}$

$\begin{array}{ll}3.4 & \text { What do we call a frontier? }\end{array}$

$\begin{array}{ll}3.5 & 122\end{array}$

$4 \quad$ Data analysis $\quad 122$

4.1 Expectile estimation for different periods 122

$\begin{array}{lll}4.2 & \text { Performance estimation } & 123\end{array}$

5 Discussion $\quad 127$

$6 \quad$ Acknowledgement 130

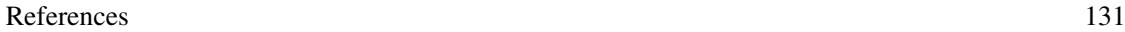




\title{
An analysis of life expectancy and economic production using expectile frontier zones
}

\author{
Sabine K. Schnabel ${ }^{1}$ \\ Paul H.C. Eilers ${ }^{2}$
}

\begin{abstract}
The wealth of a country is assumed to have a strong non-linear influence on the life expectancy of its inhabitants. We follow up on research by Preston and study the relationship with gross domestic product. Smooth curves for the average but also for upper frontiers are constructed by a combination of least asymmetrically weighted squares and $P$-splines. Guidelines are given for optimizing the amount of smoothing and the definition of frontiers. The model is applied to a large set of countries in different years. It is also used to estimate life expectancy performance for individual countries and to show how it changed over time.
\end{abstract}

\footnotetext{
${ }^{1}$ corresponding author; Max Planck Institute for Demographic Research, Rostock, Germany and Biometris, Wageningen University and Research Center, Wageningen, The Netherlands; sabine. schnabelewur.nl

${ }^{2}$ Department of Biostatistics, Erasmus Medical Center, Rotterdam, The Netherlands
} 


\section{Introduction}

It is well known that life expectancy at birth $\left(e_{0}\right)$ is higher in wealthy countries. This is supported by Figure 1. This association was first investigated by Preston (Preston 1975, 1976). There is a curvilinear relationship between period life expectancy and gross domestic product per capita (GDP). Large scatter is observed around the trend, generally wider at low GDP. A natural question to ask is whether we can estimate a "frontier", a curve of the highest attainable $e_{0}$ against GDP. Such a frontier would be of value to demographers who study groups of countries. It could also be an important tool for policy makers in individual countries in order to set goals for public health systems.

Figure 1: Average life expectancy versus gross domestic product per capita in 1990 PPP dollars. Data for the year 2000 including 126 countries. Countries with the highest GDP are the United States, Luxembourg and Norway.

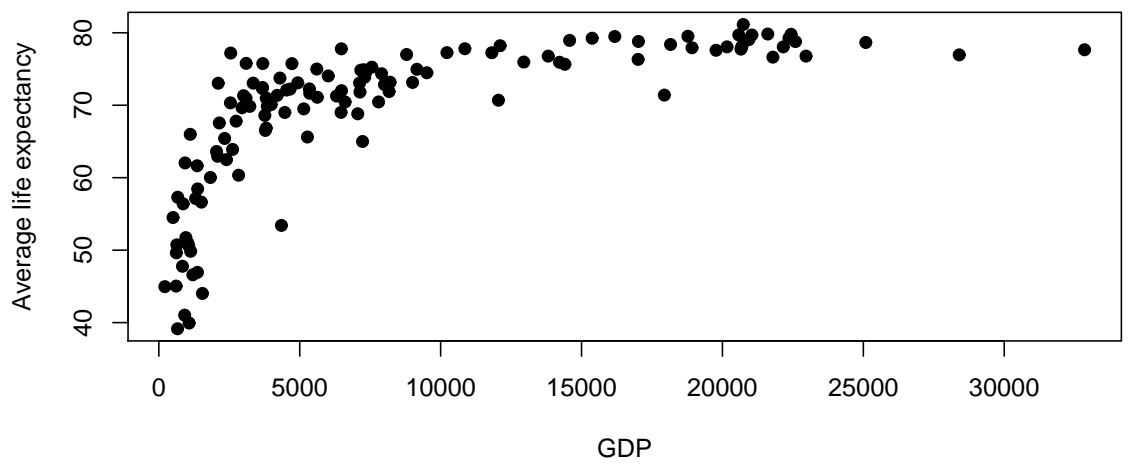

In this article we propose a new methodology for smooth frontier estimation, and we apply it to recent and historical data on GDP and period life expectancy for a large group of countries. We propose this frontier approach as a generalization of Preston's model. This framework is an extension of an established smoothing method for trend estimation. Whereas the original method gives equal weights to data points above and below a trend curve, we use asymmetric weights. If we increase the weights of the points above the curve, the curve will be drawn upward. Strong asymmetry leads to the desired frontier 
curve. The principle we use is known as asymmetric least squares or least asymmetrically weighted squares $\left(\mathrm{LAWS}^{3}\right)$.

In this paper we will use the word frontier in a soft interpretation, i.e., as a frontier zone that is located in the upper part of the data. Most data points will be located under the estimated curve, but some of the observation might also be found above the curve due to sampling variation and other factors. Therefore, in the following, the use of the word frontier is equivalent to a frontier zone and not used as an absolute boundary.

Smoothing with LAWS is a simple and easily implemented idea. It also poses new and interesting challenges. One is the choice of the optimal amount of smoothing. We show that ideas from mixed model technology can be applied fruitfully for this purpose, leading to an automatic choice of the amount of smoothing. A second problem is the choice of the amount of asymmetry that defines the frontier. We have no statistical answer to that, but we provide a practical rule of thumb.

The relationship between GDP and $e_{0}$ has been studied extensively. In Section 2.1 we give an overview of the literature. As far as we know, we are the first to propose and apply a general framework for frontier estimation in the context of life expectancy. In other areas, especially for frontier and efficiency estimation in econometrics, other approaches have been used. We give a short overview of them in Section 2.2. The demographic data that motivated our research is presented in detail in Section 2.3. Section 3 presents LAWS and its application to smoothing, including the determination of the optimal amount of smoothing. We also discuss the issue of removal of influential data points and outliers. We show how to compute LAWS statistics for theoretical distributions and use it to propose guidelines for setting the amount of asymmetry. Section 4 lays out the extensive application of LAWS to life expectancy data that motivated this research. The proposed method is also useful for other relations and other explanatory variables than the one presented in the example. In the final section 5 we discuss our results, desirable improvements and possible extensions.

\section{An overview of previous research}

This section offers an overview of previous research. Because of the intended demographic audience we give a presentation of what has been published on the relationship between life expectancy and gross domestic product per capita. We give less space to the literature on quantile regression and models for efficiency estimation.

\footnotetext{
${ }^{3}$ A common abbreviation is ALS, but we like to avoid it for two reasons: first, ALS is also commonly used as abbreviation of alternating least squares and second, ALS also means amyotrophic lateral sclerosis i.e. a fatal neurodegenerative disease.
} 


\subsection{Life expectancy and GDP}

Historically the development of life expectancy has been an important topic in population studies. In Bengtsson (2006) a collection of articles on the linear rise in life expectancy and its history and prospects can be found. For our investigations the contribution of (Oeppen 2006) is of special interest. It reviews life expectancy development and related research since 1820. Over decades researchers have hypothesized about the limits to life expectancy. In (Oeppen and Vaupel 2002) the authors review these hypotheses. However, the article's main finding about the development of life expectancy is that record life expectancy has been increasing linearly over the last 150 years. This measure can be considered a frontier to life expectancy and thus promotes the interest in investigating limits and appropriate methods for their estimation.

In the 1970s the demographer Samuel Preston investigated the influence of economic conditions on life expectancy (Preston 1975, 1976). For the mean curve of this relationship he used a logistic model with fixed coefficients. Three waves of data from 1900, 1930 and 1960 were used in this cross-sectional analysis. As a measure of economic performance Preston used national income per capita as the independent variable to model the mean trend. The logistic model that Preston used to describe the relationship is a restrictive assumption of the functional form of this relationship. To our knowledge Preston's work has never been formally extended to measure the frontier but focusses on describing the mean trend, although Easterlin (1996) stated that Preston's curve could be described as a production frontier of income as input and life expectancy as output.

Preston's research was reprinted 30 years later (Preston 2007a) and joined by an extensive discussion and rejoinder by the author (Preston 2007b). The contributions in the discussion stress the importance of health interventions on mortality development $(\mathrm{Ku}-$ nitz 2007), and the contribution of technical progress to population health (Bloom and Canning 2007), and discuss why the same amount of income can buy progressively more health over time (Wilkinson 2007). Riley (2007) highlights the fact that more research is needed in order to determine factors influencing life expectancy other than income alone and stresses that countries' mortality histories need more attention.

Deaton (2004) also refers to Preston's study and fits a non-parametric populationweighted regression function for the mean relationship between per capita GDP and life expectancy for the year 2000.

Rodgers (1979) suggests that there is a relationship between life expectancy at birth and income at the individual level and observes an asymptotic behaviour from empirical data. He proposes a non-linear model using the inverse, logarithm or other transformation for income as the independent variable. In his paper he continues to investigate in detail the relationship between life expectancy, income and income distribution. This contri- 
bution was reprinted in Rodgers (2002) and joined by a discussion including Wilkinson (2002), Porta, Borrell, and Copete (2002), Deaton (2002) and Lynch and Smith (2002).

Others argue that the mean level of income is not as important to life expectancy as how this money is distributed. Wilkinson also contributed to this field of research concluding that there is a lack of association between gross national product (GNP) per capita (measured in 1985 US dollars) and life expectancy in developed countries (Wilkinson 1990). He argues that with better comparability of international data in terms of GNP this association needs more research. When instead he uses purchasing power parity adjusted GNP per capita in a later paper (Wilkinson 1992) he finds a weak association between GNP per capita and combined life expectancy for the 23 OECD countries.

Becker, Philipson, and Soares (2003) investigated longevity convergence and also the relationship between income and longevity referring to and confirming Preston's study using a logarithm function for data from 1965 and 1995. They conclude that there is no convergence in income per capita in this period despite longevity convergence. This is consistent with a shift over time of the cross-sectional relationship. While the described relationship holds for the mean trend of $e_{0}$ and GDP, it has never been tested to model frontiers.

\subsection{Models for frontier estimation}

\subsubsection{Quantile regression}

Quantile regression is a popular tool for the purpose of frontier estimation. It was originally presented by Koenker and Bassett (1978) as a generalization of the linear model. Quantile regression is based on asymmetrically weighting the sum of absolute values of residuals. It estimated the conditional quantile functions of the underlying distribution. In contrast to quantile regression we propose asymmetrically weighting the sum of squared residuals that leads to so called expectiles as introduced by Newey and Powell in 1987. LAWS is based on ordinary least squares modeling and thus shares the properties and the simple concept of this approach.

\subsubsection{Econometric methods for efficiency estimation}

Questions of frontier estimation are discussed in productivity analysis, an area of econometrics. Here researchers model the production or cost frontier using so called Data Envelopment Analysis (DEA) or Stochastic Frontier Analysis (SFA). DEA is based on linear programming techniques aiming at a convex hull as explained in Charnes, Cooper, and Rhodes (1978). SFA is a popular parametric tool used to investigate productivity frontiers. It is a linear model with a two-fold error term accommodating the variation

of the frontier and the technical inefficiencies of the individual observations. The work 
of Kumbhakar and Lovell (2000) gives a comprehensive overview of SFA. Assuming a certain error distribution, it can be shown that LAWS is equivalent to SFA. This relationship can be deduced from Aigner, Amemiya, and Poirier (1976) and Newey and Powell (1987). Both DEA and SFA are used to estimate frontiers of a multiple input-output relation to measure the efficiencies of the individual observations. For example, they were used to describe the relationship between healthy life expectancy and health care status of a country in Evans et al. (2001); Hollingsworth and Wildman (2003). In addition to DEA and SFA Kokic et al. (1997) propose M-quantiles to model production frontiers. It can be shown that there is a relationship between expectiles and M-quantiles (Jones 1994). Kokic et al. also describe how to use M-quantiles to measure productive efficiency.

\subsection{Data description}

In the analysis in Section 4 we focus on the same variables as Preston and investigate the relationship between life expectancy at birth (average as well as sex-specific) and the logarithm of gross domestic product per capita (logGDPpc) expressed in 1990 purchasing power parity adjusted (PPP) Geary-Khamis dollars. PPP adjustment goes back to Karl Gustav Cassel who developed this concept in the 1920s. The Geary-Khamis method combines the concepts of PPP and average international prices. This technique was first introduced in Geary (1958) and further developed by Khamis (Khamis 1969, 1970, 1972). It is the most widely used method in this field, e.g. by the United Nations, OECD, the statistical office of the European Union and others. For our analysis we use data from 1900 to 2005 . The sample size in the waves is varying. Therefore we limit further analysis to years with at least 20 countries in the sample. This is the case for 66 years between 1900 and 2005 (1900, 1910, 1920, 1930, 1940 and every year between 1945 and 2005). Life expectancy data come from a range of sources including the HMD (Human Mortality Database 2007), national statistical offices, the Penn World tables (Heston, Summers, and Aten 2002) and the United Nations. Information on logGDPpc was also gathered from a range of sources that include the above, contributions by Maddison e.g. (Maddison 2001) and data from the Total Economy Database (The Conference Board and Groningen Growth and Development Centre 2006). Some descriptive measures of the data set can be seen in Figure 2. The data includes over 200 countries at different points in time.

In order to get a better impression of the data, it is helpful to have a closer look at an individual country and its development over time. This is a purely descriptive look at the data. In Figure 3 we plotted all data pooled over all times (average life expectancy against

$\log$ GDPpc, for 66 years) and indicated the development of life expectancy in Japan and Sweden. Japan is the current leader in life expectancy, especially for females. 
Figure 2: $\quad$ Box-plots of life expectancy of women (red) and men (blue) 19502000 in five year intervals. The number of countries available in each year is given by the sample size $n$ indicated below each boxplot.

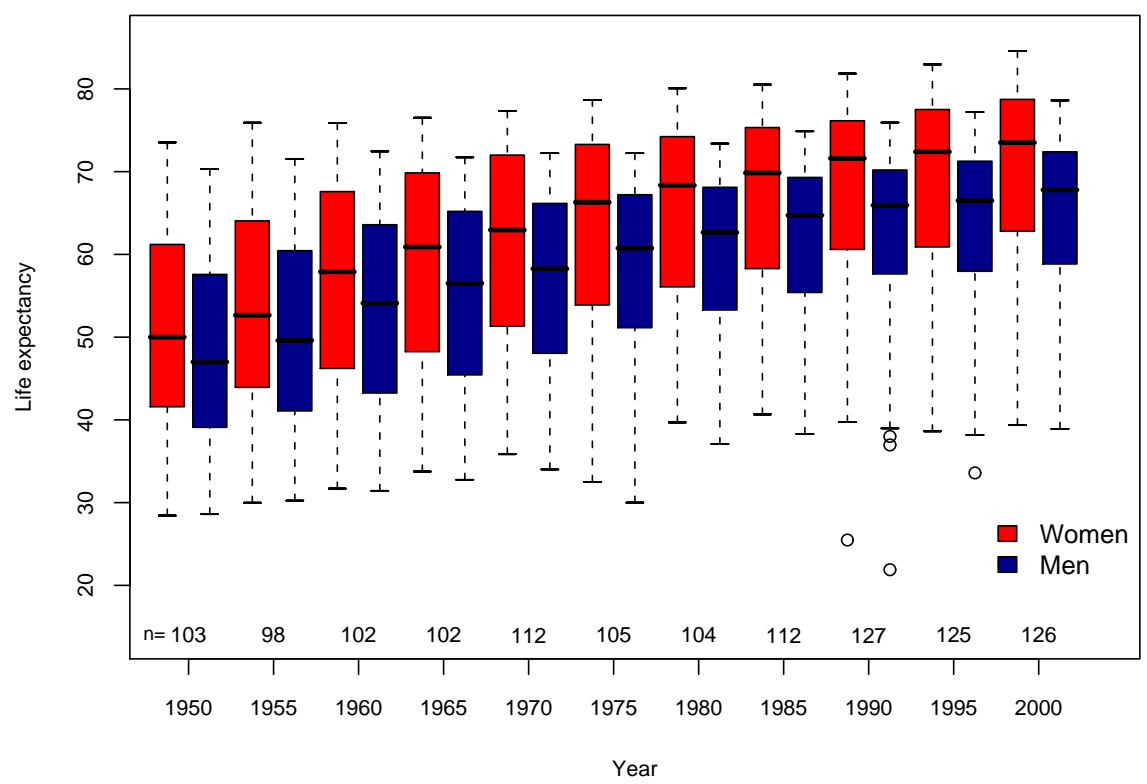

Historically, Japan experienced big gains in this measure after World War II and increasing development ever since. Sweden is a country with a long time series of data and it has always been among the countries with the highest life expectancy worldwide.

We observe different trajectories for the earlier years for the two countries. Sweden had an average period life expectancy of about 52 years in 1900 which has steadily increased to more than 80 years in 2005 . On the other hand Japan had a low average life expectancy of 37 years in 1900 and a mixed mortality history until after World War II. Then Japan experienced a rapid increase in life expectancy well up to 82 years in 2005 for the two sexes combined. 
Figure 3: $\quad$ Pooled data over all years. Development of average life expectancy over time in Japan (red circles) and Sweden (blue triangles).

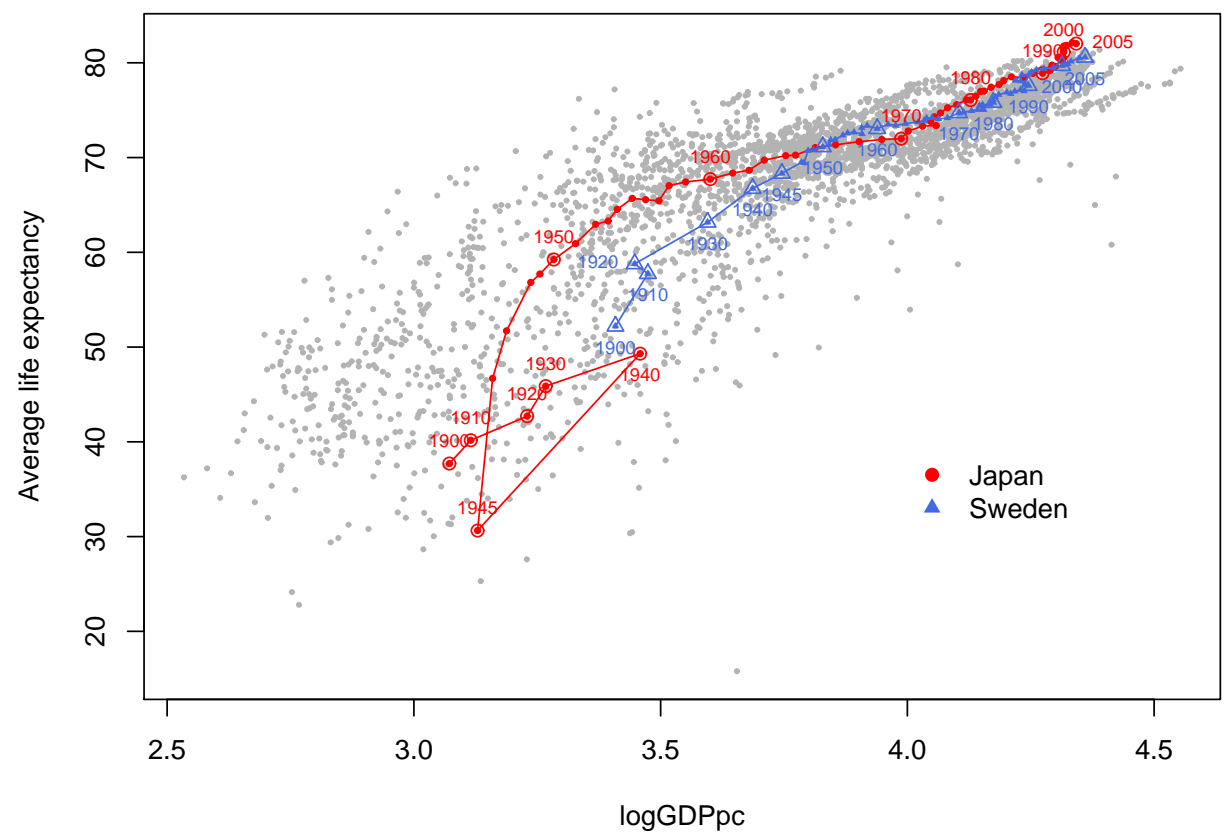

\section{Least Asymmetrically Weighted Squares Smoothing}

In the following, we propose Least Asymmetrically Weighted Squares estimation as a suitable technique for modeling frontier curves. We will apply this method to life expectancy and economic performance in Section 4. 


\subsection{LAWS in a nutshell}

In ordinary least squares (OLS) estimation one seeks to minimize the sum of squares

$$
S_{\mathrm{oLS}}=\sum_{i}\left(y_{i}-\mu_{i}\right)^{2}
$$

where $y_{i}$ is the response and $\mu_{i}$ is the estimated value. For example, in linear regression is $\mu_{i}=\hat{\beta}_{0}+\hat{\beta}_{1} x_{i}$ with $\hat{\beta}$ the estimated coefficient vector.

Least asymmetrically weighted squares estimation (LAWS) seeks to minimize the following objective function for a range of values $p, 0<p<1$ :

$$
S=\sum_{i} w_{i}(p)\left(y_{i}-\mu_{i}(p)\right)^{2}
$$

with weights

$$
w_{i}(p)= \begin{cases}p & \text { if } y_{i}>\mu_{i}(p) \\ 1-p & \text { if } y_{i} \leq \mu_{i}(p)\end{cases}
$$

where $y_{i}$ is the response variable and $\mu_{i}(p)$ is the estimated value according to a statistical model. The obtained functions $\mu(p)$ are called p-expectiles as introduced by Newey and Powell in 1987. LAWS is a weighted version of ordinary least squares where the weights depend on the sign of the residuals. When estimating an expectile, we fix a value $p$ between 0 and 1. For an upper frontier we may choose e.g. $p=0.98$. In the iterative fitting procedure each point $i$ of the data set is assigned a weight $w_{i}(p=0.98)$ according to (2). This means a point located above the estimated curve $\mu(0.98)$ receives a weight $w_{y_{i}>\mu_{i}(0.98)}=0.98$. A data point below or on the estimated curve is assigned weight $w_{y_{i} \leq \mu_{i}(0.98)}=0.02$. OLS estimation is a special case of LAWS for $p=0.5$. It is extremely easy to fit any LAWS model: simply iterate between weighted regression and re-compute the weights. The objective function is convex, so a unique minimum is guaranteed.

For simplicity, we suppress the dependence on $p$ in the following notation.

When implementing a flexible functional form of the expectile, we combine LAWS with $P$-splines (Eilers and Marx 1996): $\mu_{i}=\sum_{j} b_{i j} a_{j}$, where $B=\left[b_{i j}\right]$ is the matrix of $B$-spline basis functions and $a$ the coefficient vector. A penalty tunes the smoothness. Thus we are seeking to minimize the penalized LAWS function:

$$
S^{*}=(y-B a)^{T} W(y-B a)+\lambda\left\|D_{d} a\right\|^{2}
$$

with respect to coefficient vector $a . D_{d}$ is a matrix that forms $d$-th order differences of $a$. 
The model parameters are computed iteratively according to

$$
\hat{a}=\left(B^{T} \widetilde{W} B+P\right)^{-1} B^{T} \widetilde{W} y
$$

with current weights in $\widetilde{W}=\operatorname{diag}(\tilde{w})$ and the penalty $P=\lambda D_{d}^{T} D_{d}$.

To optimize the smoothing parameter $\lambda$ we can use so called leave-one-out crossvalidation. The idea is to remove each observation $\left(x_{i}, y_{i}\right)$ in turn, predict it $\left(\mu_{-i}\right)$ from the remaining ones and measure prediction performance by the cross validation score $C V=\frac{1}{n} \sum_{i=1}^{n}\left(y_{i}-\mu_{-i}\right)^{2}$. The asymmetric variant includes the weights according to (2):

$$
A C V=\frac{1}{n} \sum_{i} w_{i}\left(y_{i}-\mu_{-i}\right)^{2}=\frac{1}{n} \sum_{i} \frac{w_{i}\left(y_{i}-\mu_{i}\right)^{2}}{\left(1-h_{i i}\right)^{2}},
$$

where we have used the fact that $y_{i}-\mu_{-i}=\frac{y_{i}-\mu_{i}}{1-h_{i i}}$ with the hat matrix $H=\left(h_{i j}\right)_{i, j=1, \ldots, n}$ defined as

$$
H=W^{\frac{1}{2}} B\left(B^{T} W B+P\right)^{-1} B^{T} W^{\frac{1}{2}} .
$$

We search for the minimum $A C V$ for a range of values of $\lambda$ on a grid (linear for $\log \lambda$ ). This is done separately for every $p$. In the $A C V$ we assume that the weight vector is invariant to single missing observations. Simulation studies show that this assumption holds for more than $99 \%$ of the considered cases.

Alternatively, using the formal equivalence between penalized least squares smoothing and mixed models (Pawitan 2001; Lee, Nelder, and Pawitan 2006), we have that the smoothing parameter $\lambda=\frac{\sigma^{2}}{\tau^{2}}$, with $\sigma^{2}$ the variance of the weighted errors and $\tau^{2}$ the variance of the contrasts $D a$. We estimate these variances by

$$
\hat{\sigma}^{2}=\frac{(y-\mu)^{T} W(y-\mu)}{n-\mathrm{ED}}, \quad \hat{\tau}^{2}=\frac{\|D a\|^{2}}{\mathrm{ED}}
$$

where $n$ is the sample size and $\mathrm{ED}=\operatorname{trace}(H)$ the effective model dimension. We iterate between smoothing asymmetrically with $\lambda$, estimating variances (and a new $\lambda$ ) until convergence (in $\lambda$ and ideally also in weights). This is a variant of Schall's (1991) method for generalized linear mixed models.

In the application in Section 4 we use optimal smoothing according to Schall's algorithm. This technique gives very similar results to the use of cross-validation criteria, but due to its iterative set-up it is computationally less intensive, particularly with large data sets.

For details of the model, a simulation study, and comparison between optimal smoothing judged by cross-validation criteria versus the use of Schall's algorithm, we refer to Schnabel and Eilers (2009b). 


\subsection{Extreme observations}

In order to improve the quality and robustness of the estimation, outlier detection is commonly used in regression methods. This can be also done in the model framework presented above. We make use of the hat matrix from the estimation procedure in order to identify outliers or so-called extreme observations. For linear regression (Hoaglin and Welsch 1978) suggested as a rule of thumb those points with

$$
h_{i i}>\frac{2 \mathrm{ED}}{n}
$$

to qualify for an extreme point, i.e. an influential observation. In our context we use the hat matrix $H$ of the model as defined in (3) and for $n$ the respective sample size of the analyzed wave. We suggest that the points which were commonly classified as extreme observation for at least 80 of the 99 expectiles with $0.01 \leq p \leq 0.99$ (in increments of $0.01)$ are excluded from the analysis and the entire set of expectiles re-estimated. In order to further check for extreme observations in the vertical direction we suggest checking for candidates against the mean curve for $p=0.5$. As a rule of thumb, a point $i$ will be classified as an extreme observation in the vertical direction if

$$
\left|y_{i}-\mu_{i}(0.5)\right|>3 \mathrm{SD}
$$

as a rule of thumb. SD is the empirical standard deviation of the residuals. We propose to remove the detected points to improve the data quality.

With this methodology, we propose a statistical criterion for judging about potential extreme observations and their removal from the data set. However, in any application it might be also useful to exclude other observations from the data under consideration based on particularities of the data, data quality or other arguments.

\subsection{Theoretical expectiles}

Expectiles can also be computed for theoretical distributions. Assume we have a probability density function $f(x)$ with

$$
F(x)=\int_{-\infty}^{x} f(u) d u \quad \text { and } \quad G(x)=\int_{-\infty}^{x} u f(u) d u .
$$

Here $F(x)$ is the distribution function, i.e. the cumulative density, and $G(x)$ is the partial moment function. The theoretical expectile is denoted by $e_{p}$. In expectile estimation we 
seek to minimize (1) with weights according to (2). For a continuous distribution this is equivalent to

$$
\min _{e_{p}}(1-p) \int_{-\infty}^{e_{p}}\left(u-e_{p}\right)^{2} f(u) d u+p \int_{e_{p}}^{\infty}\left(u-e_{p}\right)^{2} f(u) d u
$$

Minimizing this form leads to

$$
(1-p) \int_{-\infty}^{e_{p}}\left(u-e_{p}\right) f(u) d u+p \int_{e_{p}}^{\infty}\left(u-e_{p}\right) f(u) d u=0
$$

After some algebra and replacement for $F(x)$ and $G(x)$ we can determine the theoretical $p$-expectile $e_{p}$ by

$$
e_{p}=\frac{(1-p) G\left(e_{p}\right)+p\left(m-G\left(e_{p}\right)\right)}{(1-p) F\left(e_{p}\right)+p\left(1-F\left(e_{p}\right)\right)}
$$

with $m$ the mean of the underlying distribution $F$ and $G(\infty)=m$. Solving for $p$, with $z=e_{p}$, we get

$$
p=\frac{G(z)-z F(z)}{2(G(z)-z F(z))+(z-m)} .
$$

This relationship follows from (Newey and Powell 1987; Jones 1994). According to Theorem 1 in the latter article, expectiles shift and scale like expected values with changes in mean $m$ and standard deviation $\sigma$ of $f(x \mid m, \sigma)$.

In order to determine the theoretical expectiles of a distribution, we choose a sensible grid for $z$ and compute the corresponding vector of $p$ s by numerical inversion.

\subsection{What do we call a frontier?}

In the methodological context of this paper we do not use the notion of frontier in an absolute way but as a frontier zone. Contrary to an upper envelope of the data, we employ a rather soft definition of a frontier, i.e. a curve that is located above most of the data, but some points may exceed this soft frontier due to random variation. This concept of a "soft" frontier is consistent with the notions in the literature of efficiency analysis using Stochastic Frontier Analysis e.g. in Kumbhakar and Lovell (2000) (as detailed in Section 2.2.2). A frontier as the upper envelope is more consistent with the concept of Data Envelopment Analysis based on linear programming. This approach is not pursued here. 
In the framework of expectile estimation it is up to the user what to choose as an upper frontier in terms of parameter $p$.

As seen above it is possible to estimate the expectile at almost any $p \in(0,1)$. In the context of frontier estimation we are looking to identify a suitable frontier for the given relationship. Therefore we have to decide on an appropriate value for $p$. We may use a common distribution as the reference distribution, e.g. the standard normal distribution. According to (4) in the standard normal case we have $p$ as

$$
p=\frac{\phi(z)+z \Phi(z)}{2 \phi(z)+2 z \Phi(z)-z}
$$

with $\phi, \Phi$ the respective density and distribution function of the standard normal distribution $N(0,1)$. Choosing $z$ equidistant in $[-3,3]$ covers values of $p$ in almost the entire range from 0 to 1 .

It is up to the user to decide which value of $p$ to choose for a frontier to be estimated. With the help of Table 1 the user can get a better insight into what a certain value for $p$ would be in terms of the normal distribution as a reference. In our opinion 2 standard normal deviations (or $p=0.9958$ ) might be a good choice for a frontier. However, this is a subjective choice for the user. The use of a reference distribution also allows for performance comparison in terms of standard deviations.

Table 1: $\quad p$ expressed in standard deviations from the mean of a standard normal distribution.

\begin{tabular}{lll}
\hline standard deviation & \multicolumn{1}{c}{$p$} & cumulative density \\
\hline $\boldsymbol{0}$ & $\mathbf{0 . 5}$ & 0.5 \\
$\mathbf{0 . 5}$ & 0.7791 & 0.6915 \\
0.5531 & $\mathbf{0 . 8}$ & 0.7099 \\
0.8577 & $\mathbf{0 . 9}$ & 0.8045 \\
$\boldsymbol{1}$ & 0.9286 & 0.8413 \\
1.1383 & $\mathbf{0 . 9 5}$ & 0.8725 \\
$\mathbf{1 . 4 7 4 9}$ & $\mathbf{0 . 9 8}$ & 0.9299 \\
$\mathbf{1 . 5}$ & 0.9812 & 0.9332 \\
$\mathbf{1 . 7 1 5 4}$ & $\mathbf{0 . 9 9}$ & 0.9569 \\
$\boldsymbol{2}$ & 0.9958 & 0.9772 \\
$\mathbf{2 . 5}$ & 0.9992 & 0.9938 \\
$\mathbf{3}$ & 0.9999 & 0.9987 \\
\hline
\end{tabular}


Note that the reference distribution is being used here only as a guide for selecting values of $p$. It is possible and useful to compare the empirical expectiles with theoretical expectiles of the reference distribution, but we do not pursue this approach here.

\subsection{Individual performance measures}

Kokic et al. (1997) propose M-quantiles to model production frontiers and to measure productive efficiency. According to Jones (1994) there is a relationship between expectiles and M-quantiles. From previous applications in this area (see Hollingsworth and Wildman 2003) as well as from the theoretical connections we suggest efficiency analysis in the context of LAWS. As a variant of the definition suggested in Kokic et al. (1997) we assign as value for the so called performance the value $p$ of the closest $p$-expectile (in terms of absolute distance):

$$
\operatorname{performance}\left(x_{i}\right)=\min _{p} \arg \left|y_{i}-\mu_{i}(p)\right| .
$$

In order to receive a wide range of possible performance values in a sample we estimate a dense grid of $p$-expectiles. In the analysis in Section 4.2 we applied this performance measure to data on life expectancy and GDP in an investigation of sex-specific reactions to improvement in the economic performance of a single country, and include as well comparisons between countries.

This relative performance measure is an approximation on the grid of used $p$ 's. It will be possible to develop a technique that helps us to find the exact $p$-expectile for a given data point.

\section{Data analysis}

\subsection{Expectile estimation for different periods}

In this section we present results from the analysis of selected waves. We chose the years 1950, 1960 and 2000 (with sample sizes of 101, 99 and 126 countries respectively). After estimating a set of expectiles - namely 99 curves with $0.01 \leq p \leq 0.99$ - we proceeded by searching for extreme points in the data sets in order to improve the estimated curves according to the methodology presented in Section 3.2. We removed the points which were classified as extreme and re-estimated the set of expectiles. In Figure 4 on page 124 the results after detecting extreme observations are presented with the influential points depicted in grey circles. We removed three data points from the 1950, 1960 and 2000 data. The removed points in 1950 and 1960 corresponded mostly to countries in the Persian Gulf area that showed a GDP inflated by profits from oil trading and comparably 
low life expectancy for the level of nominal wealth. After removing these observations the estimated expectiles improved. This can be seen in Figure 5 where we present the estimated set of expectiles for 1950 before and after removing extreme observations. It has also been pointed out in (Yee 2008) that expectiles are more susceptible to outliers than the more robust quantile estimation. This supports our approach to combine outlier detection with estimating expectiles.

We chose the years 1950 and 1960 in order to see potential $e_{0}$ increases associated with increases in GDP following the post-war economic boom. Range, variance and composition of the three data sets differ. This leads to different characteristics in the estimated curves. The spread of the estimated expectiles is smaller in 1960 than in 1950. However, the mean distance between $e_{0.9}$ and $e_{0.1}$ is about the same. The smaller spread of expectiles is seen again in 2000. The expectiles confirm the hypothesis of longevity convergence by Becker, Philipson, and Soares (2003) and are in line with their statement of no obvious convergence in GDP, as in the three years under consideration, the standard deviation for GDP is largest in 2000.

\subsection{Performance estimation}

While in Figure 3 we plotted the development of life expectancy over time of individual countries in a purely descriptive way, in the following we present plots that can be used to illustrate the performance of individual countries, as introduced in Section 3.5. Estimated frontiers and therefore also performance values depend on the composition of the sample in the respective waves. As mentioned above, data on 200 countries were available at different points in time. However, the maximum sample size in the analysis below is 127 countries. Only complete observations in both $e_{0}$ and GDP can be used. The heterogeneity of the sample needs further consideration. Only data of 10 countries is present in all 66 waves (i.e. data are available and included in the analysis). These are mainly developed countries that have recognized good data quality. Nevertheless, the countries represented in the years multiple of 5 is a heterogeneous mix of geographical areas, stage of development and population size. In the data set used for our analysis, some information on low GDP countries were excluded due to lack of data. All performance values are relative to the respective sample and not to be interpreted as absolute performance values. 
Schnabel \& Eilers: An analysis of life expectancy and economic production using expectile frontier zones

Figure 4: $\quad$ Average life expectancy versus logGDPpc. Selected expectiles for three waves: $1950,1960,2000$.

$\mathrm{p}=0.01,0.02,0.05,0.10,0.20,0.50,0.80,0.90,0.95,0.98,0.99$. Colors from red to blue with increasing $p$. Extreme observations indicated in grey.
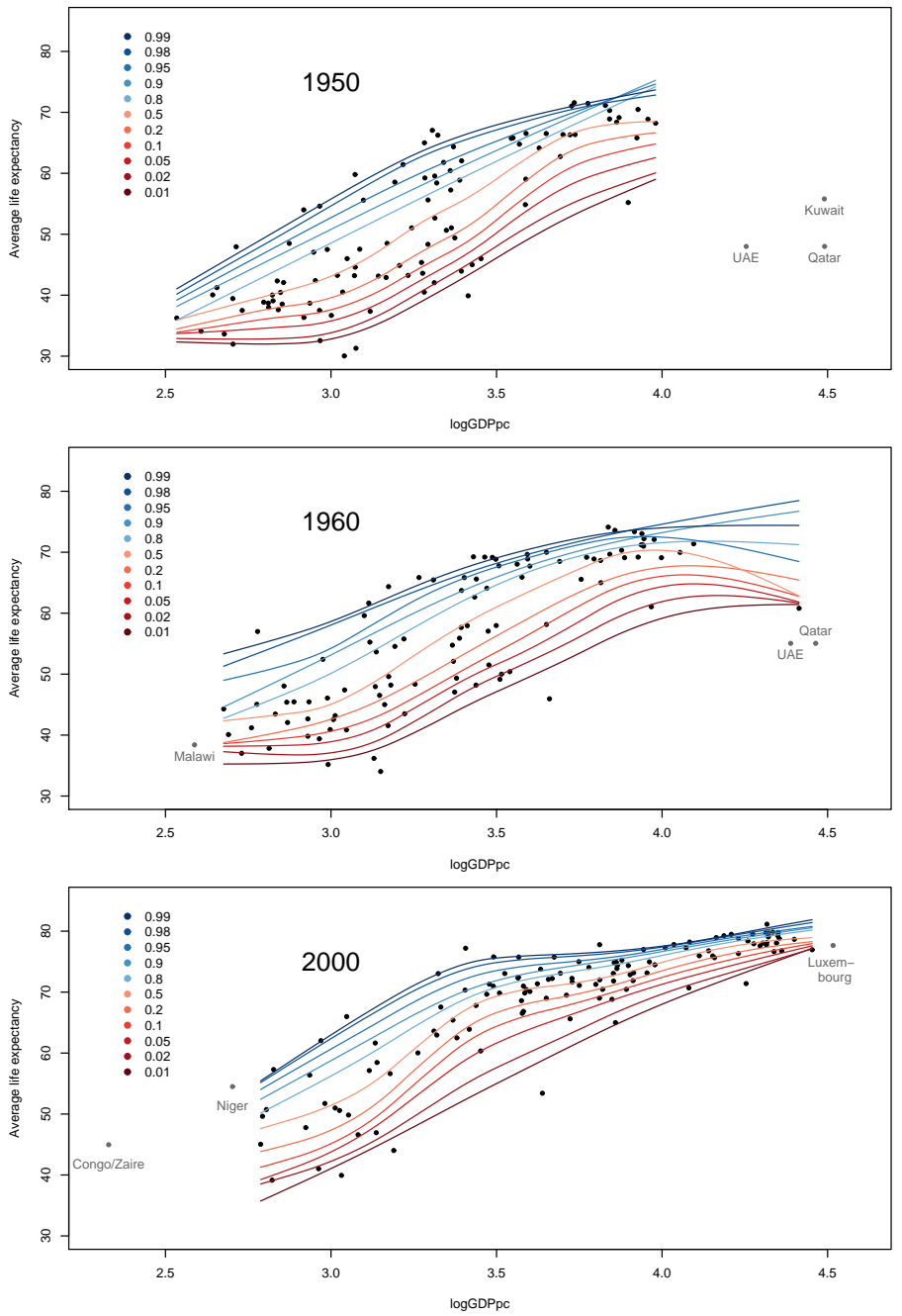
Figure 5: $\quad$ Data for 1950. Left panel: estimated curves for all data; right panel: re-estimated curves after removing three extreme observations (grey triangles).
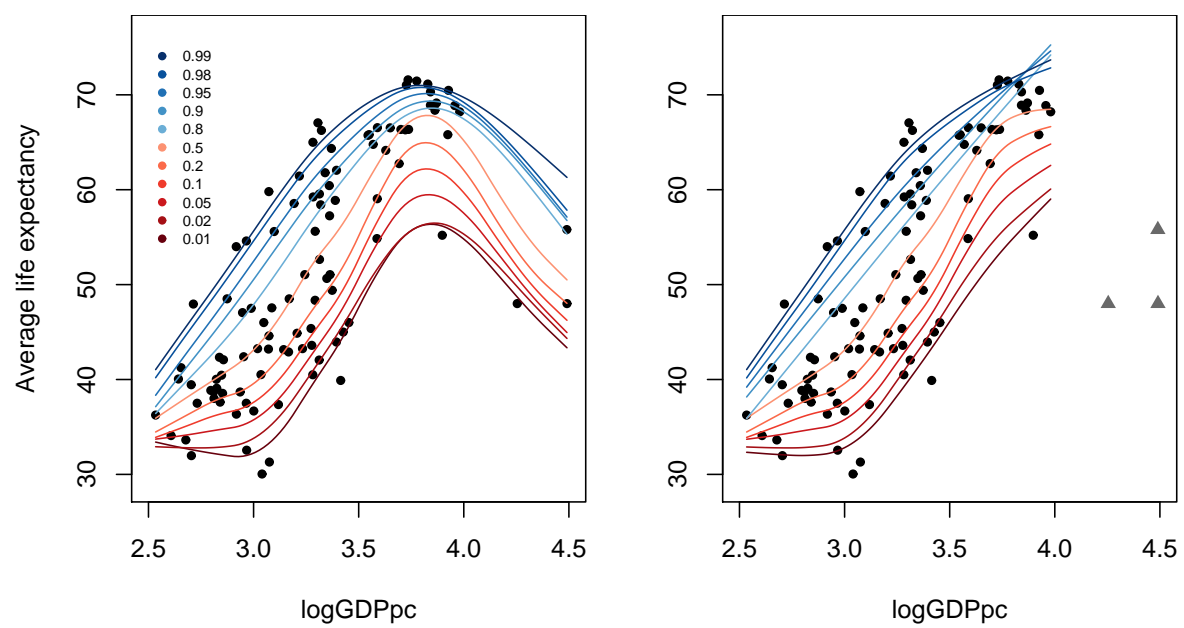

In Figure 6 we plot the relative performance values according to (5) for Sweden and Japan in 1900-2005. As already seen in Figure 3, Japan has a mixed mortality history. This is also true for its relative performance, as its values stretch the entire range between 0.01 and 0.99 . The median performance for the displayed years is $p=0.86$; in about $30 \%$ of the displayed years Japan is closest to the highest estimated frontier curve (with $p=0.99$ ). Japan has been in the top group in terms of life expectancy, economic level and relative performance in the last 25 years. In comparison, Sweden historically always had a high level of relative performance. The series of $p$ covers the range from 0.79 to 0.99 . The median performance is $p=0.97$ and in more than half of the shown years relative performance $p$ is at least 0.95 . Figure 6 shows the scale for relative performance $p$ and a scale in standard deviations from the mean of the standard normal distribution as a reference distribution.

With our data set it is possible to analyze data for females and males separately and to have a look at possible sex-specific differences in the development of life expectancy. This analysis is motivated by the hypothesis that an increase in GDP translates differently into life expectancy gains for males and females. This is especially observed after the second World War when women show a higher increase in life expectancy while GDP increases. 
Figure 6: $\quad$ Relative performance of Sweden and Japan (average life expectancy versus $\operatorname{logGDPpc}$ ) on a scale in standard deviations from the mean of a standard normal distribution (left) and a scale of $p$ (right).

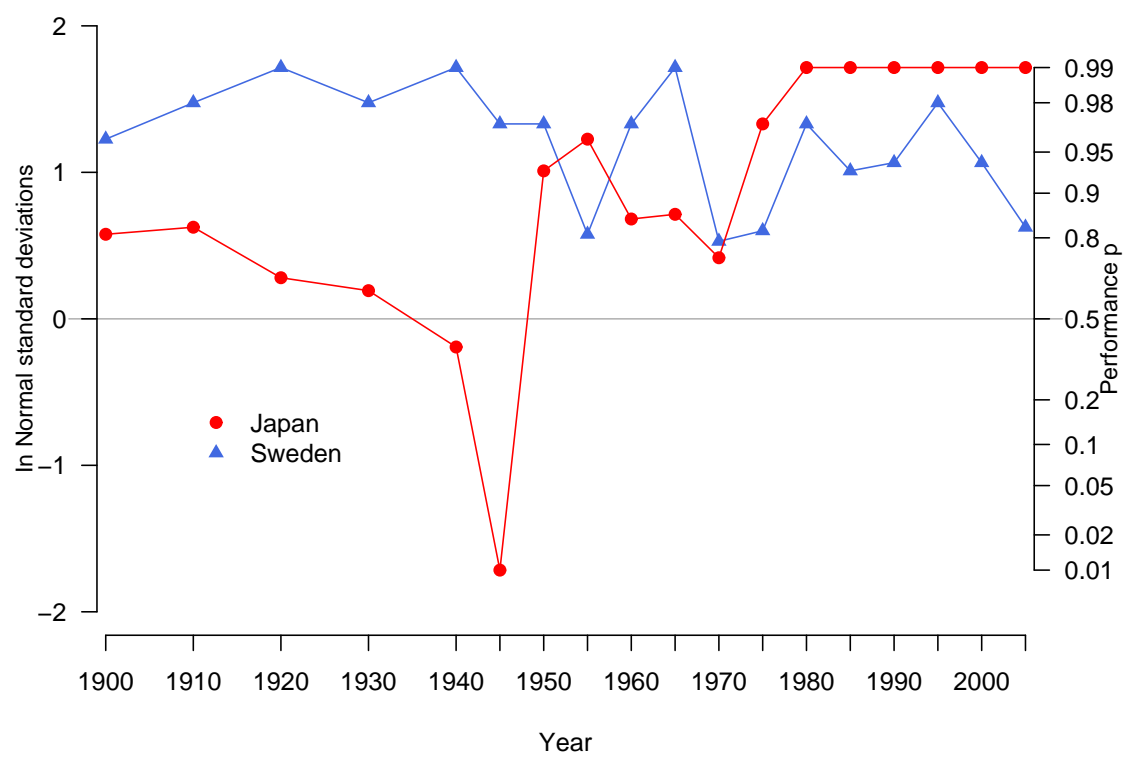

In Figure 7 we depict sex-specific relative performance for four European countries: France, Finland, Germany and the Netherlands.

Historically France has been a country with big gaps between female and male life expectancy. This gender gap in life expectancy stays quite constant during the period covered by our data set. On average women lived more than seven years longer than men. Moreover, female life expectancy lies near higher frontiers in the analysis than males. In addition to the gender gap in life expectancy alone, we find a similar gap in performance between the two groups. Finland shows a similar pattern in sex-specific life expectancy differences and differences in relative performance. Finnish women lived on average more than 7.5 years longer than Finnish men from 1900 to 2005. As the figure makes clear, the 
gap in relative performance is also persistent over time. Germany is the third country in this example. We notice that both sexes are very similar in terms of relative performance, with the level increasing in recent years. The Netherlands is an example of a country with a rapid decline in relative performance over the last 30 years, with the exception of the most recent period. This is especially pronounced for females.

Figure 8 on page 129 depicts the case of Denmark. We present the relative performance of males and females in the lowest graph. The other two graphs show the life expectancy of Danish males and females along with the estimated expectiles at Denmark's value of GDP for the respective year. The set of three graphs helps to relate the relative performance values to the absolute level of $e_{0}$ and gives a good impression of the spread in expectiles. The gender gap in life expectancy in Denmark widened considerably from 1950 to 1980 , increasing from 3 to a peak of more than 6.5 years. This is followed by a decline to a level of less than 5 years. Females also seem to have systematically lower relative performance values and show a drop in performance during and beyond the period where the gap between female and male life expectancy widened. The causes of stagnating Danish life expectancy, particularly for females, have been explored in (Fiig Jarner, Masotti Kryger, and Dengsøe 2008).

\section{Discussion}

We developed and applied a new statistical framework to model the relationship between GDP and life expectancy. The core idea is to use least asymmetrically weighted squares (LAWS) to explore not only the central tendency but also extreme regions of the conditional distribution. LAWS leads to a very simple iterative estimation algorithm; it is easily combined with $P$-splines ( $B$-splines with a discrete roughness penalty) to model trends and frontiers. By borrowing ideas from mixed models, the weight of the penalty can be optimized straightforwardly. The result is a powerful asymmetric smoother. We compared it to quantile smoothing splines and observed our smoother to be superior (Schnabel and Eilers 2009b).

The smoother was applied to large samples of countries, at different points in recent history. Not only did we compute trends and frontier curves, we also computed performance measures, a kind of "relative LAWS ranking" to quantify how well each country did over time. We present time series for a number of countries.

Comparison of results over time is influenced by the changes in sample composition. Relative performance is always relative to the sample. In order to overcome this problem and enable better comparability over time, a model including time and GDP will be needed. 
Figure 7: $\quad$ Comparison between female and male relative performance for four countries: a scale in standard deviations from the mean in a standard normal distribution (left) and a scale of relative performance $p$ (right).
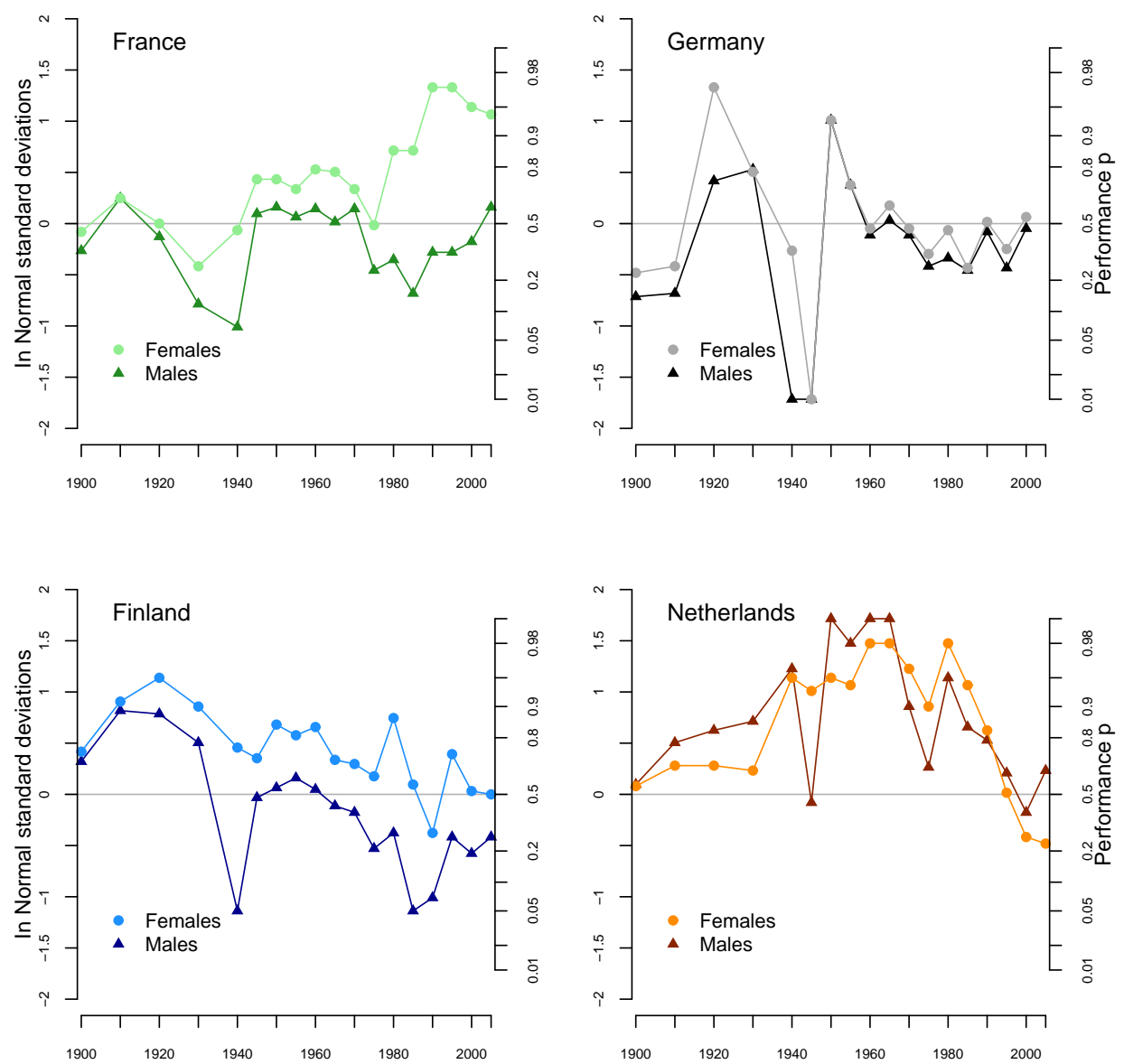
Figure 8: $\quad$ Relative performance of Danish males and females (lowest panel). For comparison, estimated expectiles at the respective GDP indicated in the top panels.
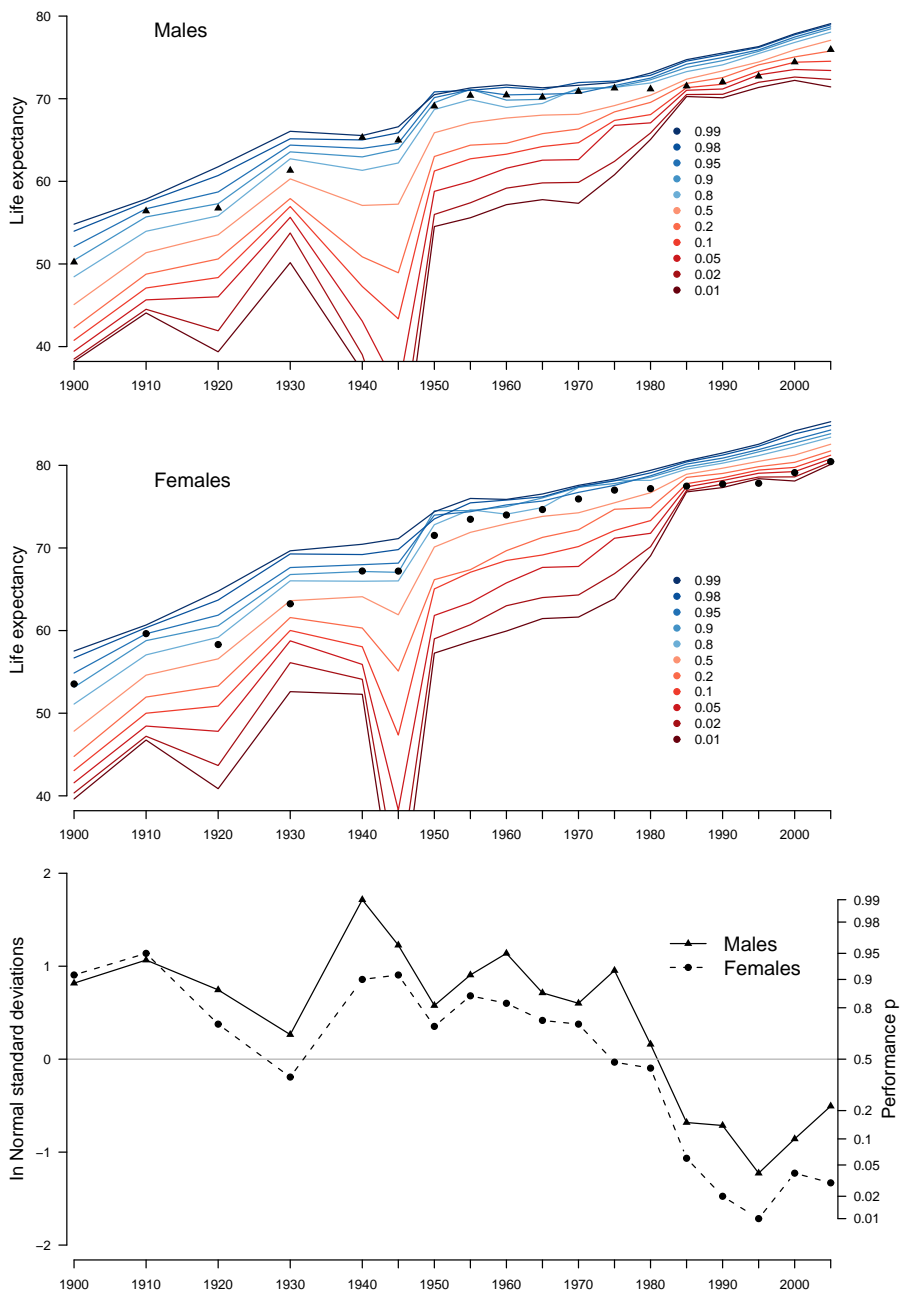
What is a frontier? We believe it is a matter of convention, comparable to choosing a 95th or 98th percentile. The LAWS equivalent of quantiles is expectiles. Unfortunately, statisticians and applied scientists as yet have little "feel" for expectiles. We show how to compute expectiles for theoretical distributions and use the results to relate expectiles to familiar $z$-scores from the standard normal distribution.

We are convinced that our asymmetric smoother is a valuable tool for demographers and policy makers, because it allows one to visualize and quantify life expectancy (performance). The computations are fast. Our software is written for the R system (R Development Core Team 2008) and we will be happy to share it.

A serious problem remains in quantile estimation as well as in expectile estimation: the curves can cross. Theoretically this is impossible, but due to sampling variation it is common in practice. Because the LAWS curves are computed in isolation (for each different value of the asymmetry parameter $p$ ), neighboring curves are not taken into account in the estimation procedure, and cross-overs can occur. In the literature one can find several proposals for creating non-crossing (smooth) quantile curves. In principle these apply to LAWS too. Our experiments indicate that a bilinear or "bundle" model is a promising alternative: $\mu(x, p)=a(x) g(p)+b(x)$, where $\mu(x, p)$ is the $p$-expectile curve, $b(x)$ is a trend, $g(p)$ an asymmetry function and $a(x)$ represents the local width of the expectile bundle. Further details and first results can be found in Schnabel and Eilers (2009a).

\section{Acknowledgement}

This work was done while the first author was at the Max Planck Institute for Demographic Research in Rostock (Germany). We are especially grateful to James E. Oeppen from the Max Planck Institute for Demographic Research in Rostock (Germany) for providing the data set that has formed the basis of our analysis. Furthermore we also thank Jutta Gampe for helpful comments and discussions as well as two anonymous reviewers for their constructive remarks that helped improve our paper. 


\section{References}

Aigner, D.J., Amemiya, T., and Poirier, D.J. (1976). On the estimation of production frontiers: maximum likelihood estimation of the parameters of a discontinuous density function. International Economic Review 17(2): 377-396. doi:10.2307/2525708.

Becker, G.S., Philipson, T.J., and Soares, R.R. (2003). The quantity and quality of life and the evolution of world inequality. National Bureau of Economic Research. (NBER Working Paper Series 9765).

Bengtsson, T. (2006). Perspectives on Mortality forecasting - III. The linear rise in life expectancy: history and prospects. No. 3 in Social Insurance Studies. Swedish National Social Insurance Agency.

Bloom, D.E. and Canning, D. (2007). Commentary: The Preston Curve 30 years on: still sparking fires. International Journal of Epidemiology 36(3): 498-499. doi:10.1093/ije/dym079.

Charnes, A., Cooper, W.W., and Rhodes, E. (1978). Measuring the efficiency of decision units. European Journal of Operational Research 2(6): 429-444. doi:10.1016/03772217(78)90138-8.

Deaton, A. (2002). Commentary: The convoluted story of international studies of inequality and health. International Journal of Epidemiology 31(3): 546-549. doi:10.1093/ije/31.3.546.

Deaton, A. (2004). Health in an age of globalization. In: C. Graham and S.M. Collins (eds.) Brookings Trade Forum 2004 : Globalization, Poverty, and Inequality. Brookings Institution Press: chap. Globalization and inequality: 83-130.

Easterlin, R.A. (1996). Growth triumphant: the twenty-first century in historical perspective. The University of Michigan Press.

Eilers, P.H.C. and Marx, B.D. (1996). Flexible smoothing with B-splines and penalties. Statistical Sciences 11(2): 89-121. doi:10.1214/ss/1038425655.

Evans, D.B., Tandon, A., Murray, C.J.L., and Lauer, J.A. (2001). Comparative efficiency of national health systems: cross national econometric analysis. British Medical Journal 323: 307-310. doi:10.1136/bmj.323.7308.307.

Fiig Jarner, S., Masotti Kryger, E., and Dengsøe, C. (2008). The evolution of death rates and life expectancy in Denmark. Scandinavian Actuarial Journal 2: 147-173. doi:10.1080/03461230802079193.

Geary, R.C. (1958). A note on the comparison of exchange rates and purchasing power 
between countries. Journal of the Royal Statistical Society: Series A 121(1): 97-99. doi: $10.2307 / 2342991$.

Heston, A., Summers, R., and Aten, B. (2002). Penn World Table Version 6.1. Center for International Comparisons at the University of Pennsylvania (CICUP). (Database).

Hoaglin, D.C. and Welsch, R.E. (1978). The hat matrix in regression and ANOVA. The American Statistician 32(1): 17-22. doi:10.2307/2683469.

Hollingsworth, B. and Wildman, J. (2003). The efficiency of health production: re-estimating the WHO panel data using parametric and non-parametric approaches to provide additional information. Health Economics 12(6): 493-504. doi:10.1002/hec.751.

Human Mortality Database (2007). [electronic resource]. University of California, Berkeley (USA) ; Max-Planck-Institut für demografische Forschung, Rostock (Germany). http://www.mortality.org or http://www.humanmortality.de.

Jones, M.C. (1994). Expectiles and M-quantiles are quantiles. Statistics \& Probability Letters 20(2): 149-153. doi:10.1016/0167-7152(94)90031-0.

Khamis, S.H. (1969). Neoteric index numbers. Calcutta: Indian Statistical Institute. (Technical report).

Khamis, S.H. (1970). Properties and conditions for the existence of a new type of index numbers. Sankhya: The Indian Journal of Statistics: Series B 32: 81-98.

Khamis, S.H. (1972). A new system of index numbers for national and international purposes. Journal of the Royal Statistical Society: Series A 135(1): 96-121. doi: $10.2307 / 2345041$.

Koenker, R. and Bassett, G.W. (1978). Regression quantiles. Econometrica 46(1): 33-50.

Kokic, P., Chambers, R., Breckling, J., and Beare, S. (1997). A measure of production performance. Journal of Business \& Economic Statistics 15(4): 445-451. doi: $10.2307 / 1392490$.

Kumbhakar, S.C. and Lovell, C. (2000). Stochastic Frontier Analysis. Cambridge University Press.

Kunitz, S.J. (2007). Commentary: Samuel Preston's "The changing relation between mortality and level of economic development". International Journal of Epidemiology 36(3): 491-492. doi:10.1093/ije/dym076.

Lee, Y., Nelder, J.A., and Pawitan, Y. (2006). Generalized linear models with random effects - unified analysis via H-likelihood. Chapman \& Hall/CRC. 
Lynch, J. and Smith, G.D. (2002). Commentary: Income inequality and health: The end of the story? International Journal of Epidemiology 31: 549-551. doi:10.1093/ije/31.3.549.

Maddison, A. (1995). Monitoring the world economy 1820-1992. OECD Development Center.

Maddison, A. (2001). The world economy: a millennial perspective. OECD Development Center.

Newey, W.K. and Powell, J.L. (1987). Asymmetric least squares estimation and testing. Econometrica 55(4): 819-847. doi:10.2307/1911031.

Oeppen, J. (2006). Life expectancy convergence among nations since 1820: separating the effects of technology and income. In: T. Bengtsson (ed.) Perspectives on mortality forecasting - III. The linear rise in life expectancy: history and prospects. Försäkringskassan, Swedish Social Insurance Agency: 55-82.

Oeppen, J. and Vaupel, J.W. (2002). Broken limits to life expectancy. Science 296(5570): 1029-1031. doi:10.1126/science.1069675.

Pawitan, Y. (2001). In All Likelihood: Statistical modelling and inference using likelihood. Oxford University Press.

Porta, M., Borrell, C., and Copete, J.L. (2002). Commentary: Theory in the fabric of evidence on the health effects of inequalities in income distribution. International Journal of Epidemiology 31(3): 543-546. doi:10.1093/ije/31.3.543.

Preston, S.H. (1975). The Changing Relation between Mortality and Level of Economic Development. Population Studies 29(2): 231-248. Reprinted in the International Journal of Epidemiology Vol. 36, pp.484-490, 2007.

Preston, S.H. (1976). Mortality patterns in national populations. With Special Reference to Recorded Causes of Death. Academic Press.

Preston, S.H. (2007a). The Changing Relation between Mortality and Level of Economic Development - Reprint. International Journal of Epidemiology 36(3): 484-490. doi:10.1093/ije/dym075.

Preston, S.H. (2007b). Response: On "The changing relation between mortality and level of economic development". International Journal of Epidemiology 36: 502-503.

R Development Core Team (2008). R: A language and environment for statistical computing. [electronic resource]. Vienna, Austria: R Foundation for Statistical Computing. Http://www.R-project.org. 
Riley, J.C. (2007). Commentary: Missed opportunities. International Journal of Epidemiology 36(3): 494-495. doi:10.1093/ije/dym078.

Rodgers, G.B. (1979). Income and inequality as determinants of mortality: an international cross-section analysis. Population Studies 33(2): 343-351. doi: $10.2307 / 2173539$.

Rodgers, G.B. (2002). Income and inequality as determinants of mortality: an international cross-section analysis (reprint). International Journal of Epidemiology 31: 533-538.

Schall, R. (1991). Estimation in Generalized Linear Models with random effects. Biometrika 78(4): 719-727. doi:10.1093/biomet/78.4.719.

Schnabel, S.K. and Eilers, P.H.C. (2009a). Non-crossing smooth expectile curves. In: J.G. Booth (ed.) Proceedings of the 24th International Workshop on Statistical Modellung in Ithaca. Cornell University.

Schnabel, S.K. and Eilers, P.H.C. (2009b). Optimal expectile smoothing. In press at Computational Statistics and Data Analysis. doi:10.1016/j.csda.2009.05.002.

The Conference Board and Groningen Growth and Development Centre (2006). Total Economy Database. [electronic resource]. Http://www.ggdc.net.

Wilkinson, R.G. (1992). Income distribution and life expectancy. British Medical Journal 304: 165-168. doi:10.1136/bmj.304.6820.165.

Wilkinson, R. (2002). Commentary: Liberty, fraternity, equality. International Journal of Epidemiology 31(3): 538-543. doi:10.1093/ije/31.3.538.

Wilkinson, R.G. (1990). Income distribution and mortality: a 'natural' experiment. Sociology of Health \& Illness 12(4): 391-412. doi:10.1111/j.1467-9566.1990.tb00079.x.

Wilkinson, R.G. (2007). Commentary: The changing relation between mortality and income. International Journal of Epidemiology 36(3): 492-494. doi:10.1093/ije/dym077.

Yee, T.W. (2008). vgam family functions for quantile regression. [electronic resource]. Http://www.stat.auckland.ac.nz/ yee. 